\title{
Simple preparation and characterization of nickel ferrite nanocrystals by a thermal treatment method.
}

\begin{abstract}
Nickel ferrite nanocrystals were prepared from an aqueous solution containing metal nitrates and poly (vinyl pyrrolidone) (PVP) as a capping agent. To stabilize the particles, they were thermally treated at various temperatures from 623 to 823 . K at which calcination occurred, thereby stabilizing the particles, controlling the growth of the nanoparticles, preventing their agglomeration, and creating a uniform distribution of particle sizes. The characterization studies were conducted by X-ray diffraction (XRD), Fourier transform infrared spectroscopy (FT-IR), and transmission electron microscopy (TEM). The crystallization was completed between 723 and 823. K, as revealed by the absence of organic absorption bands in the FT-IR spectra Magnetization measurements were obtained at room temperature by using a vibrating sample magnetometer (VSM), which showed that the calcined samples exhibited ferromagnetic behaviors. Finally, we used TEM images and FT-IR spectra to investigate the same process in the absence of PVP and with various of concentrations of PVP for comparison with the results acquired from using the optimum concentration that was used in this work.
\end{abstract}

Keyword: Nickel ferrite; Nanocrystals; Thermal treatment; Magnetization measurements. 